\title{
Treatment of gastric cancer in the older population
}

\author{
Jakub Kenig, Piotr Richter \\ Department of General, Gastrointestinal, Oncologic Surgery and Transplantology, I Chair of General Surgery, \\ Jagiellonian University Medical College, Krakow, Poland
}

\begin{abstract}
Gastric cancer (GC) is predominantly a disease of the elderly as approximately $60 \%$ of all patients are 70 years of age or older. At present, there are no guidelines dedicated to this group, and current treatment strategies are mainly based on evidence from clinical trials often carried out on younger patients.

The GC in older patients is typically located in the distal third of the stomach and it is well/moderately differentiated, having mainly an intestinal type of tumor by Lauren's criteria. Lymph nodes and peritoneal metastases have been reported less frequently in comparison to younger patients.

Older patients are a very heterogeneous population in terms of co-morbidity, physical reserve, cognitive function, and social support. Treatment side effects can cause more serious problems than cancer itself, so the comprehensive geriatric assessment (CGA) is as important as the cancer staging. Chronological age alone is not a contraindication for treatment. Surgery is the preferred standard treatment option for resectable GC. However, the prognostic significance of surgery and other treatment options is unknown in the frail group. Fitter patients, according to the CGA, should qualify for the same treatment as younger patients. Frail patients should be discussed during oncogeriatric meetings. Surgery, the benefits of limitation of the surgical resection, and no or non-selective lymphadenectomy should all be analyzed. In experienced hands, minimal invasive surgery is favorable in the short- and long-term. In cases of severe frailty, the best supportive care can often be the best option.
\end{abstract}

Key words: older patients, gastric cancer, frailty

The prevalence of gastric cancer (GC) has gradually decreased over the last decades, nevertheless, it remains a major cause of cancer-related death and remains the fifth most common cancer in the world. GC is also predominantly a disease of the elderly as approximately $60 \%$ of all patients are 70 years and older. Subsequently, an aging population means that the number of older patients with GC is increasing continuously [1].

Surgery is the preferred standard treatment option for resectable GC. However, the prognostic significance of surgery and other treatment modalities is still unknown in frail patients. At present, there are also no guidelines dedicated to older patients and current treatment strategies are mainly based on evidence from clinical trials frequently carried out on younger patients [2]. Therefore, surgeons and oncologists experience many difficulties when making decision in this age group. Very often these decisions are taken based on comorbidity burden, subjective assessments or are age driven.

\section{The clinical and pathological characteristics of GC in older patients}

There is a male predominance among older patient with GC, which contrasts with younger patients where the gender ratio is typically closer to 1:1. There is usually no family history. The GC is typically located in the distal third of the stomach and it is

\section{How to cite:}

Kenig J, Richter P. Treatment of gastric cancer in the older population. NOWOTWORY J Oncol 2021; 71: 245-250.

This article is available in open access under Creative Common Attribution-Non-Commercial-No Derivatives 4.0 International (CC BY-NC-ND 4.0) license, allowing to download articles and share them with others as long as they credit the authors and the publisher, but without permission to change them in any way or use them commercially. 
well/moderately differentiated. Moreover, histologically, older patients present mainly with an intestinal type of tumor by Lauren's criteria. It frequently metastasizes to the liver. Lymph nodes and peritoneal metastases have been reported less frequently in comparison to younger patients [3].

According to the Japanese classification of GC, the predominant type of early gastric cancer (EGC) in older patients is the superficial depressed type Ilc, followed by the superficial elevated type lla, and the polypoid type I [4]. Genetically, older patients have more frequent TP-53 and HER2 overexpression, and more microsatellite instability-high tumors [5].

\section{Preoperative assessment and treatment decisions}

As was mentioned in our previous publications, the population of older patients is very heterogeneous in terms of co-morbidity, physical reserve, cognitive function, and social support [6]. Current routine pre-operative assessment also cannot adequately identify patients at risk. Therefore, the comprehensive geriatric assessment (CGA) was introduced to help determine the primary status of the older patient, to diagnose frailty syndrome (surrogate of the biological age), and to identify how to optimize the patient's condition before the start of the treatment. Many older adults have unidentified, uncommunicated, and therefore unaddressed aging-related conditions that are associated with morbidity and early mortality $[7,8]$. The natural life expectancy of older people is surely shorter than that of younger people. Considering their limited remaining lifetime, their postoperative quality of life is as valuable as the need to cure or remove the cancer. Therefore, the International Society of Geriatric Oncology (SIOG) and The European Society for Medical Oncology (ESMO) recommends the use of the CGA to determine biological age before the beginning of the treatment.

In general, based on the CGA, we can differentiate three groups of older patients:

1. Fit: patients without any deficits in the CGA domains and less than 80 years old. In this group, standard oncologic treatment can be offered and the postoperative outcomes are comparable with younger patients.

2. Pre-frail: patients with one or two deficits in the CGA domains or more than 80 years old. In these patients, rehabi- litation should be recommended to improve resilience to surgical stress by, at least, augmenting functional capacity and nutritional status before surgery.

3. Frail patients: patients with three or more impaired domains in the CGA or 80 years old with two deficits in the CGA. A tailored approach should be discussed in a geriatric multidisciplinary team meeting [9].

It is also possible to determine the severity of the frailty using a cumulative deficit model for the CGA [10].

\section{Treatment of gastric cancer in older patients}

The therapeutic options for GC depending on the cancer stage $[11,12]$ including options for frailer, older patients, are presented in table I.

Patients with early GC (T1) can undergo endoscopic resection using an endoscopic submucosal dissection (ESD) technique. The ESD, in experienced hands, is an effective and safe procedure for older patients, with clinical and oncological outcomes comparable to younger population. A higher prevalence of cardiopulmonary problems was reported during the procedure when compared to the younger group. However, they were managed effectively during the procedure without further clinical sequelae. Therefore, the ESD should be the standard treatment for management of early GC fulfilling the Japanese criteria of a negligible risk of lymph node metastasis for both fit and frail patients $[13,14]$.

At present, surgical resection is the main curative treatment option for patients with gastric cancer GC stage T1 b and higher. However, it can cause high morbidity and mortality, particularly in the older population. Improvement in anesthesiology, standardization of the surgical technique, and perioperative care improve the 30-day outcomes significantly in comparison to previously reported data. Katai et al. and Zhou et al. reported that surgery can be safely performed with an excellent prognosis in older patients with GC [15, 16]. In contrast, Fujisaki et al. observed that after gastrectomy there was as higher rate of postoperative non-surgical complications (pneumonia, heart failure, and liver dysfunction) in comparison to younger patients [17]. In turn, Wakahara et al. observed a 10\% increase in the overall postoperative morbidity, including the rate of anastomotic leakage that was significantly elevated in the older group [18]. Mengardo et al. concluded that $\geq 80$ years of age is

Table I. Therapeutic options for gastric cancer depending on the cancer stage [11, 12], including options for frail, older patients

\begin{tabular}{|lll}
\hline Stage factors & Fit patients & Frail patients \\
\hline T1N0 & - endoscopic resection & endoscopic resection \\
& - limited resection & - best supportive care in severe frailty \\
\hline T2-4 N0-2 & - preoperative chemotherapy, followed by total/subtotal & - subtotal/partial gastrectomy with no or selected \\
& - gastrectomy and postoperative chemotherapy & lymphadenectomy \\
& purgery followed by adjuvant chemotherapy & best supportive care in severe frailty \\
not-resectable metastatic & - palliative treatment & best supportive care
\end{tabular}


a negative independent factor impacting overall survival (OS), thus, these patients should be carefully selected for surgery [19]. Endo et al. reported on a heterogeneous population of patients aged 85 years who underwent surgery for gastric cancer. This group had a better prognosis than those who did not undergo surgery. Females, patients aged 85-89years, and patients with stage IB-IIIC cancer had significantly better OS with surgery than without. For males, patients aged 90 years of age, or stage IA patients, the decision to perform surgery should be carefully discussed, and best support care may be an optimal strategy [20]. Most of the postoperative deaths in these patients were due to pneumonia and not due to GC. However, the most important limitation of the study is that the authors divided the patients based on their chronological age alone and not on the presence of frailty factors (a surrogate of biological age). Therefore, I would view the group of 90-year-old patients in this study as being equivalent to a severe frailty group.

To conclude, it appears that fit and mildly frail patients can and should be operated on with acceptable short-term outcomes. In the case of severe frailty, surgery might not be the optimal option. However, currently studied patients were fit older patients mostly defined based on their chronological age and/or comorbidity burden and not on the biological age. We are still lacking good data on the long-term outcomes of frail patients, because their risk of mortality after hospitalization remains particularly high during the first 6 months. Moreover, surgery is a well-known trigger for postoperative institutionalization and dependency on other people in older patients. Therefore, quality of life as an end point is even more important than OS or disease-free survival (DFS).

The next question is the extent of the surgery. Total/subtotal gastrectomy with a D2 lymphadenectomy is recommended for most fit patients with resectable advanced GC as a standard surgical procedure. In the case of older patients, the essential clinical question is whether perioperative trauma can be limited which, in turn, may reduce the risk of postoperative morbidity/ mortality and increase the quality of life. Therefore, there is a trend among surgeons to perform a subtotal gastrectomy, since a total gastrectomy in this age group had been associated with higher rates of postoperative morbidity and mortality. A Dutch study reported that older patients qualified to total gastrectomy had a relative risk of 2.15 for in-hospital mortality and 3.25 for morbidity, as compared to those who had undergone a partial gastrectomy [21]. Similarly, Katai et al. demonstrated that total gastrectomy in octogenarian patients was associated with higher operative and 90-day mortality [22]. Moreover, the 5 -year overall survival was better in older patients in partial as opposed to total gastrectomy ( $86 \%$ vs. 67\%). There are also studies showing the benefit to limit the resection to specific margins in the case of cancers with beneficial histology [23].

To conclude, the extent of the resection, if there is any possibility, should be limited in older patients. However, similarly as above, we do not have good studies using biological age as opposed to chronological.

Limited data are also available to clarify the survival benefit of D2 lymphadenectomy for older patients. Shinozuka et al. analyzed 3484 patients from many centers who received surgical resection for GC. The authors selected patients aged $\geq 80$ years with T2-4 GC. Their performed propensity score matching to balance the essential variables (stage of disease and gastrectomy type). The D2 group had significantly longer operative times, more blood loss, and more retrieved lymph nodes than the non-D2 group. The D2 group had a greater incidence of intra-abdominal abscesses (grade $\geq 1$ in the Clavien-Dindo classification) than the non-D2 group. The overall, disease-specific and relapse-free survival rates of the D2 group tended to be worse than those of the non-D2 group (hazard ratios: 1.49, 1.70 and 1.14, respectively). The non-D2 group had a slightly longer relapse-free survival compared with that of the D2 group, indicating that limited lymphadenectomy did not increase the risk of disease recurrence [24].

Also essential, is that postoperative complications after gastrectomy influence the prognosis. Wang et al. and Kanda et al. showed that morbidity following gastrectomy shortens the long-term survival of older patients with GC; D2 lymphadenectomy was an independent risk factor of postoperative complications [25-27]. In turn, Takeda et al. recommend that standard radical lymph node dissection should be used for tumors extending through the serosa (T3) and/or involving extragastric lymph nodes (N2), even in patients aged 80 years or more [28].

To conclude, it seems that in fit (based on the CGA) older patients a standard D2 lymphadenctomy can be carried out. However, the more severe the frailty, the more selective the lymphadenectomy should be. Larger prospective studies are required to clarify the necessity of D2 lymph node dissection to treat older, frail patients.

Most of the studies on minimal invasive surgery in older patients with the GC report have comparable oncological results and good short-/long-term outcomes both in unmatched and propensity-matched patients aged 80 years and older [29]. Total gastrectomy, a Charlson comorbidity index $\geq 4$, and pathological N stage were identified as independent prognostic factors for overall survival in patients undergoing a laparoscopic gastrectonmy [30]. The first Western experience in laparoscopic distal gastrectomy was published by Rausei S. et al. this year, including 46 patients aged 80 years and older. The authors concluded that the laparoscopic approach reduces the effect of surgical trauma without compromising the oncological results [31]. There are also the first studies analyzing frailty as a prognostic factor in the laparoscopic group of older patients with GC. Tanaka et al. reported that operative complications (especially systemic complications) were positively associated with an increase in clinical frailty scores. Moreover, the overall 5-year survival rate and the 5-year survival rates 
for those with a clinical frailty score of $1-2,3-4$, and $5-7$ were respectively $70.9 \%, 59.8 \%$, and $35.1 \%$. Therefore, the authors conclude that frailty has a great impact on operative morbidity and prognosis in the elderly, and the CFS score could be a promising prognostic predictor, especially for frail patients with advanced gastric cancer [32].

To conclude, minimal invasive gastrectomy has the potential to provide a balance between oncological clearance and quality of life issues that remain crucially important in the older population. However, full a comprehensive geriatric assessment was not performed in any of the studies. The legal point of view is also interesting. Older cancer patients are offered a standardized treatment model geared toward younger adults by their physicians, due to the fear of being accused of undertaking the incorrect oncological treatment. In this context, it may be useful to surgeons to highlight the Polish Supreme Court verdict from September 24, 2015 (V CSK 738/14 - the extent of obligation to provide information by physicians), discussed in the article by dr. Radosław Drozda from the Department of Forensic Medicine at the Wroclaw Medical University. It concluded that "the choice between alternative treatment methods belongs to the patient, and the clinician should present the patient with all available treatment options that are possible in their physical condition - at most with an indication as to which of these options is the most beneficial according to the doctor..." and "... it is the patient - despite a lack of medical training - who should make the ultimate decision on the surgical method that they will be subjected to. The role of the physician is to convince the patient why (and for what medical reasons) it might be worth undergoing a riskier procedure. The patient however has the right (driven by personal reasons or even superstition) to pick a method that would be less invasive and is likely to have a lower efficacy than the method proposed by the clinician" [33].

\section{Perioperative chemotherapy}

Due to high recurrence rates, multimodal treatment is a standard for GC in stage IB disease and higher. However, concerns remain regarding chemotherapy in older patients due to the risks of perioperative morbidity from toxicity. Therefore, to determine the feasibility of treating patients over the age of 65 , a predefined exploratory subgroup analysis of patients within the randomized phase II FLOT 65+ trial compared patients treated with perioperative FLOT (5-fluorouracil, oxaliplatin and docetaxel) or FLO (without docetaxel). In the study, a high level of adherence among older patients was observed: $85 \%$ of patients received all 4 preoperative cycles of FLOT, and there was no clinically significant increase in grade 3-4 toxicity postoperatively. Mortality and morbidity rates were comparable to other trials, including patients across all ages. The authors concluded that neoadjuvant FLO or FLOT chemotherapy is therefore a reasonable option in older patients with locally advanced resectable gastric cancer [34-36]. It must be stressed that, although, there was no upper age limits included in the study, the patients were generally fit. In turn, Slagter et al. evaluated treatment-related toxicity, treatment compliance, surgical complications, and event-free survival in older ( $>70$ years) versus younger ( $<70$ years) patients who underwent perioperative treatment for GC. 788 patients with resectable gastric cancer were randomized before the start of any treatment, and received preoperative chemotherapy (3 cycles of epirubicin, cisplatin or oxaliplatin and capecitabine), followed by surgery, followed by either postoperative chemotherapy or chemoradiotherapy (45 Gy, cisplatin, capecitabine). During preoperative chemotherapy, $77 \%$ of older adults versus $62 \%$ of younger adults experienced severe toxicity $(p<0.001)$ and older adults received significantly lower relative dose intensities for all chemotherapeutic drugs. Equal proportions of older and younger adults underwent curative surgery $(80 \%$ vs. $81 \%$ ), with comparable postoperative complications and postoperative mortality; $64 \%$ of older patients and $78 \%$ of young patients started adjuvant chemotherapy $(p<0.001)$. There was no difference in the severe toxicity rate between the groups; however, older adults received significantly lower relative dose intensities for all chemotherapeutic drugs [37]. The question as to whether this kind of treatment can be proposed to frail patients with good short- and long-term outcomes still remains unanswered.

In the case of patients with resected gastric cancer who have not received preoperative chemotherapy, adjuvant chemotherapy is recommended [38]. However, adjuvant chemotherapy is, generally, less well tolerated than preoperative chemotherapy. Therefore, the latter may be the preferential option. An interesting study was published this year by Schendel et al. including 75-year and older patients with GC from Canada. The 5 year DFS for the surgery only group was $67.3 \%$ and for the multimodality group was $52.9 \%(p=0.25)$. The 5 year OS for the surgery only group was $38.9 \%$ and for the multimodality group was $47.1 \%(p=0.52)$. The authors concluded that even with surgery alone, selected older patients with non-metastatic gastric cancer can obtain prolonged survival, despite not receiving standard of care multimodality therapy [39].

Concluding, limited data on these topics are available from the Western world. Most of the studies were conducted in Asia and the applicability of these results in Europe remains uncertain, not to mention the evaluation of frailty based on the comprehensive geriatric assessment.

\section{Palliative treatment}

Currently, there is little evidence of the role of palliative resection in GC in older patients. The REGATTA trial including patients with incurable gastric cancer, randomizing them to palliative chemotherapy alone or to gastrectomy with chemotherapy, showed no survival advantage of surgery, with the median OS at 16.6 months (95\% Cl: 13.7-19.8) with chemotherapy versus 14.3 months (95\% Cl: 11.8-16.3) with surgery and chemotherapy. 
The included patients were young, between 49 and 67 years old. It seems that palliative gastrectomy should not be considered in patients with advanced gastric cancer, unless there are other indications (bleeding or obstruction) [40].

As far as palliative chemotherapy is concerned, in patients over 70, it is recommended to consider tailored treatment based on biological age with two/three-drug chemotherapy regimens and dose reduced therapy.

\section{Conclusions}

Chronological age should not be a contraindication for multimodal radical treatment in older patients. The frailty (surrogate of the biological age) evaluation should be the basis for the discussion on treatment planning. At present, it is one of the most reliable factors predicting functional decline in different organs, making it more difficult for older patients to overcome surgical stress. Moreover, the potential benefits of surgery for frail patients with GC must be explored in the context of their shorter life expectancy compared to younger patients.

Therefore, before treatment begins, the following questions should be discussed:

- Is the currently planned treatment strategy correct? Are there alternative treatment options?

- What is the result of the comprehensive geriatric assessment? Can frailty syndrome be diagnosed in the patient?

- What is the risk of complications?

- What would the patient's lifespan be without treatment?

- What are the goals, preferences, and expectations of the patient? What effect might the treatment have on these goals?

- Is it possible to improve the patient's state prior to the surgical procedure?

Fit patients, according to the comprehensive geriatric assessment, should be qualified for the same treatment as younger patients. Prefrail patients should undergo pre rehabilitation and be reevaluated. Frail patients should be discussed in the oncogeriatric meeting. In the case of surgical treatment, the limitation of the resection's extent, no or selective lymphadenectomy should be considered. In experienced hands, minimal invasive surgery may be beneficial regarding the short- and long-term outcome. In the case of severe frailty, best supportive treatment can be the optimal option. The goal of the modifications is to reduce surgical stress. In older patients (aged 75 years or older), the pathological outcome and postoperative complications are predictors of survival, whereas pathological outcome and chemotherapy are predictors of survival in the younger population (aged 74 years or less). Thus, the prevention of postoperative morbidity may contribute to improved prognosis for older patients with gastric cancer [41].

However, we still need better designed studies on a larger group of patients using frailty evaluation. Existing studies on this topic are limited, too small, and lack important details with satisfactory statistical clout. In clinical observational studies, overall survival is usually considered the gold standard endpoint because it is simple and reliable to measure. Overall survival could be diluted by non-cancer-related deaths, especially in the older population. Therefore, cancer-specific survival and relative survival should be used in this group $[42,43]$. Moreover, novel endpoints should be explored such as patients reported outcomes to establish appropriate treatment guidelines for frail, older patients.

Conflict of interest: none declared

\author{
Jakub Kenig \\ Jagiellonian University Medical College \\ I Chair of General Surgery \\ Department of General, Oncologic, Gastrointestinal Surgery and \\ Transplantology \\ ul. Jakubowskiego 2 \\ 30-688 Kraków, Poland \\ e-mail:jkenig@cm-uj.krakow.pl
}

Received: 4 Jul 2021

Accepted: 6 Jul 2021

\section{References}

1. Rawla P, Barsouk A. Epidemiology of gastric cancer: global trends, risk factors and prevention. Prz Gastroenterol. 2019; 14(1): 26-38, doi: 10.5114/pg.2018.80001, indexed in Pubmed: 30944675.

2. Gaitonde S, Fischer T, Jones $M$, et al. Young age, disparities, and survival in gastric adenocarcinoma: Implications for screening strategies. J Clin Oncol. 2017; 35(4 suppl): 17-17, doi: 10.1200/jco.2017.35.4 suppl.17.

3. Joharatnam-Hogan N, Shiu KK, Khan K. Challenges in the treatment of gastric cancer in the older patient. Cancer Treat Rev. 2020; 85: 101980, doi: 10.1016/j.ctrv.2020.101980, indexed in Pubmed: 32065879.

4. Fujiya T, Endoh K, Mikuni J, et al. Clinicopathological Characteristics and Surgical Outcome of Gastric Carcinoma with Regard to Aging. Jpn J Gastroenterol Surg. 1997; 30(7): 1699-1705, doi: 10.5833/jjgs.30.1699.

5. Wu CW, Chen MH, Huang KH, et al. The clinicopathological characteristics and genetic alterations between younger and older gastric cancer patients with curative surgery. Aging. 2020; 12(18): 18137-18150, doi: 10.18632/aging. 103627.

6. Kenig J. Oncogeriatrics (part 1.). Frailty in older adults with cancer. Nowotwory. Journal of Oncology. 2019; 69(2): 55-57, doi: 10.5603/ njo.2019.0010

7. Loh KP, Soto-Perez-de-Celis E, Hsu T, et al. What Every Oncologist Should Know About Geriatric Assessment for Older Patients With Cancer: Young International Society of Geriatric Oncology Position Paper. J Oncol Pract. 2018; 14(2): 85-94, doi: 10.1200/JOP.2017.026435, indexed in Pubmed: 29436306.

8. Klabunde $\mathrm{CN}$, Ambs A, Keating NL, et al. The role of primary care physicians in cancer care. J Gen Intern Med. 2009; 24(9): 1029-1036, doi: 10.1007/s11606-009-1058-x, indexed in Pubmed: 19597893.

9. Grodzicki T, Kenig J. Problemy okołooperacyjne u osób w wieku podeszłym. PZWL Wydawnictwo Lekarskie, Warszawa 2018.

10. Kenig J, Szabat K. Oncogeriatrics (part 7.). Geriatric assessment for older patients with cancer. Nowotwory. Journal of Oncology. 2020; 70(4): 153-157, doi: 10.5603/njo.2020.0031.

11. Smyth EC, Verheij M, Allum W, et al. ESMO Guidelines Committee. Gastric cancer: ESMO Clinical Practice Guidelines for diagnosis, treatment and follow-up. Ann Oncol. 2016; 27(suppl 5):v38-v49, doi: 10.1093/annonc/ mdw350, indexed in Pubmed: 27664260

12. Kulig J, Wallner G, Drews M, et al. Polish Consensus on Treatment of Gastric Cancer; update 2017. Pol Przegl Chir. 2017; 89(5): 55-68.

13. Yang TC, Hou MC, Chen PH, et al. Clinical Outcomes and Complications of Endoscopic Submucosal Dissection for Superficial Gastric Neoplasms in the Elderly. Medicine (Baltimore). 2015; 94(44): e1964, doi: 10.1097/ MD. 0000000000001964 , indexed in Pubmed: 26554806.

14. Sinagra $E$, Luppino I, Messina $M$, et al. Endoscopic approach to early gastric cancer in older adults. J Geriatr Oncol. 2021; 12(1): 160-162, doi: 10.1016/j.jgo.2020.05.005, indexed in Pubmed: 32467026. 
15. Katai $H$, Sasako M, Sano $T$, et al. Gastric cancer surgery in the elderly without operative mortality. Surg Oncol. 2004; 13(4): 235-238, doi: 10.1016/j.suronc.2004.09.007, indexed in Pubmed: 15615661.

16. Zhou CJ, Chen FF, Zhuang CL, et al. Feasibility of radical gastrectomy for elderly patients with gastric cancer. Eur J Surg Oncol. 2016;42(2):303311, doi: 10.1016/j.ejso.2015.11.013, indexed in Pubmed: 26710992.

17. Fujisaki M, Shinohara T, Hanyu N, et al. Laparoscopic gastrectomy for gastric cancer in the elderly patients. Surg Endosc. 2016; 30(4): 13801387, doi: 10.1007/s00464-015-4340-5, indexed in Pubmed: 26123337.

18. Nie RC, Chen FP, Yuan SQ, et al. Evaluation of objective response, disease control and progression-free survival as surrogate end-points for overall survival in anti-programmed death-1 and anti-programmed death ligand 1 trials. Eur J Cancer. 2019; 106: 1-11, doi: 10.1016/j. ejca.2018.10.011, indexed in Pubmed: 30453169.

19. Mengardo V, Cormack OMc, Weindelmayer J, et al. Multicenter Study of Presentation, Management, and Postoperative and Long-Term Outcomes of Septegenerians and Octogenerians Undergoing Gastrectomy for Gastric Cancer. Ann Surg Oncol. 2018; 25(8): 2374-2382, doi: 10.1245/s10434-018-6543-1, indexed in Pubmed: 29868974.

20. Endo $S$, Shimizu $Y$, Ikenaga $M$, et al. Survival benefit of gastrectomy for gastric cancer in patients $\geq 85$ years old: A retrospective propensity score-matched analysis. Surgery. 2017; 161(4): 984-994, doi: 10.1016/j. surg.2016.10.012, indexed in Pubmed: 27894711.

21. Songun I, Putter H, Kranenbarg EMK, et al. Surgical treatment of gastric cancer: 15-year follow-up results of the randomised nationwide Dutch D1D2 trial. Lancet Oncol. 2010; 11(5): 439-449, doi: 10.1016/S1470-2045(10)70070-X, indexed in Pubmed: 20409751.

22. Katai $H$, Sasako $M$, Sano $T$, et al. The outcome of surgical treatment for gastric carcinoma in the elderly. Jpn J Clin Oncol. 1998; 28(2): 112-115, doi: 10.1093/jjco/28.2.112, indexed in Pubmed: 9544826.

23. Shuto K, Yamazaki M, Mori M, et al. [Partial Gastrectomy for Elderly Patients with Early-Stage Gastric Cancer]. Gan To Kagaku Ryoho. 2018; 45(13): 1824-1826, indexed in Pubmed: 30692366.

24. Shinozuka T, Kanda M, Ito S, et al. D2 lymph node dissection confers little benefit on the overall survival of older patients with resectable gastric cancer: a propensity score-matching analysis of a multi-institutional dataset. Surg Today. 2020; 50(11): 1434-1442, doi: 10.1007/ s00595-020-02021-7, indexed in Pubmed: 32451713.

25. Suzuki S, Kanaji S, Matsuda Y, et al. Long-term impact of postoperative pneumonia after curative gastrectomy for elderly gastric cancer patients. Ann Gastroenterol Surg. 2018; 2(1): 72-78, doi: 10.1002/ ags3.12037, indexed in Pubmed: 29863154.

26. Wang $\mathrm{S}, \mathrm{Xu} \mathrm{L}$, Wang $\mathrm{Q}$, et al. Postoperative complications and prognosis after radical gastrectomy for gastric cancer: a systematic review and meta-analysis of observational studies. World J Surg Oncol. 2019; 17(1): 52, doi: 10.1186/s12957-019-1593-9, indexed in Pubmed: 30885211.

27. Kanda $M$, Ito $S$, Mochizuki $Y$, et al. Multi-institutional analysis of the prognostic significance of postoperative complications after curative resection for gastric cancer. Cancer Med. 2019; 8(11): 5194-5201, doi: 10.1002/cam4.2439, indexed in Pubmed: 31353821.

28. Takeda J, Tanaka T, Koufuji K, et al. Gastric cancer surgery in patients aged at least 80 years old. Hepatogastroenterology. 1994; 41(6): 516-520, indexed in Pubmed: 7721236

29. Tanaka R, Lee SW, Imai Y, et al. Advantages of Laparoscopic Surgery for Gastric Cancer in Elderly Patients Aged Over 80 Years: A Propensity Score Matching Analysis. World J Surg. 2021 [Epub ahead of print], doi: 10.1007/s00268-021-06157-6, indexed in Pubmed: 34019135
30. Mikami R, Tanaka E, Murakami T, et al. The safety and feasibility of laparoscopic gastrectomy for gastric cancer in very elderly patients: short-and long-term outcomes. Surg Today. 2021; 51(2): 219-225, doi: 10.1007/s00595-020-02078-4, indexed in Pubmed: 32676846.

31. Rausei S, Galli F, Milone M, et al. Laparoscopic distal gastrectomy in old-old patients: the first Western experience. Updates Surg. 2021 [Epub ahead of print], doi: 10.1007/s13304-021-01063-x, indexed in Pubmed: 33900551.

32. Tanaka T, Suda K, Inaba K, et al. Impact of Frailty on Postoperative Outcomes for Laparoscopic Gastrectomy in Patients Older than 80 Years. Ann Surg Oncol. 2019; 26(12): 4016-4026, doi: 10.1245/s10434019-07640-0, indexed in Pubmed: 31359279.

33. Drozd R. Kto powinien wybierać metodę leczenia operacyjnego - pacjent czy lekarz? Chirurgia po Dyplomie. 2017; 2(12): 34-36.

34. Al-Batran SE, Homann N, Pauligk C, et al. Perioperative chemotherapy with fluorouracil plus leucovorin, oxaliplatin, and docetaxel versus fluorouracil or capecitabine plus cisplatin and epirubicin for locally advanced, resectable gastric or gastro-oesophageal junction adenocarcinoma (FLOT4): a randomised, phase 2/3 trial. Lancet. 2019; 393(10184): 1948-1957, doi: 10.1016/s0140-6736(18)32557-1.

35. Lorenzen S, Pauligk C, Homann N, et al. Feasibility of perioperative chemotherapy with infusional 5-FU, leucovorin, and oxaliplatin with (FLOT) or without (FLO) docetaxel in elderly patients with locally advanced esophagogastric cancer. Br J Cancer. 2013; 108(3): 519-526, doi: 10.1038/bjc.2012.588, indexed in Pubmed: 23322206.

36. van Hagen P, Hulshof MC, van Lanschot JJB, et al. CROSS Group. Preoperative chemoradiotherapy for esophageal or junctional cancer. $\mathrm{N}$ Engl J Med. 2012; 366(22): 2074-2084, doi: 10.1056/NEJMoa1112088, indexed in Pubmed: 22646630.

37. Slagter AE, Tudela B, van Amelsfoort RM, et al. Older versus younger adults with gastric cancer receiving perioperative treatment: Results from the CRITICS trial. Eur J Cancer. 2020; 130: 146-154, doi: 10.1016/j. ejca.2020.02.008, indexed in Pubmed: 32208351.

38. Smyth EC, Verheij M, Allum W, et al. Gastric cancer: ESMO Clinical Practice Guidelines for diagnosis, treatment and follow-up. Ann Oncol. 2016; 27(5): 38-49.

39. Schendel J, Jost E, Mah M, et al. Gastric cancer management in elderly patients: a population-based study of treatment patterns and outcomes in gastric cancer patients $\geq 75$ years from Alberta, Canada. Am J Surg. 2021; 221(4): 839-843, doi: 10.1016/j.amjsurg.2020.03.006, indexed in Pubmed: 32222273.

40. Fujitani K, Yang HK, Mizusawa J, et al. Gastrectomy plus chemotherapy versus chemotherapy alone for advanced gastric cancer with a single non-curable factor (REGATTA): a phase 3, randomised controlled trial. Lancet Oncol. 2016; 17(3):309-318, doi: 10.1016/s1470-2045(15)00553-7.

41. Ueno $D$, Matsumoto $H$, Kubota $H$, et al. Prognostic factors for gastrectomy in elderly patients with gastric cancer. World J Surg Oncol. 2017; 15(1): 59, doi: 10.1186/s12957-017-1131-6, indexed in Pubmed: 28284210.

42. Nie RC, Chen FP, Yuan SQ, et al. Evaluation of objective response, disease control and progression-free survival as surrogate end-points for overall survival in anti-programmed death-1 and anti-programmed death ligand 1 trials. Eur J Cancer. 2019; 106: 1-11, doi: 10.1016/j. ejca.2018.10.011, indexed in Pubmed: 30453169.

43. Howlader N, Ries LAG, Mariotto AB, et al. Improved estimates of cancer-specific survival rates from population-based data. J Natl Cancer Inst. 2010; 102(20): 1584-1598, doi: 10.1093/jnci/djq366, indexed in Pubmed: 20937991. 